diet including food types, frequency of consumption, quantities and cooking methods. A representative sample of 3970 and those with gallstone disease had their 7-DFDs coded by nutritionists using a computer program containing nutrient information on 11000 food and 55000 portion sizes. A cohort analysis used Cox regression to estimate sex specific HRs for developing gallstones for fifths of dietary cholesterol and trans fatty acid intake, adjusted for known risk factors: age, body mass index, energy, alcohol and physical activity in men and also parity and hormone replacement therapy use in women.

Results 177 women (mean age of diagnosis 66.5 years SD 9.5 years) and 90 men (64.2 years SD 9.2 years) developed symptomatic gallstones. In women, increased dietary cholesterol was associated with a decreased risk of disease (highest vs lowest fifth, HR $0.52,95 \%$ CI 0.31 to 0.86 , per fifth increase HR 0.83 , 95\% CI 0.74 to 0.93 , p for trend=0.002), with no effect in men (trend across fifths HR $0.92,95 \%$ CI 0.78 to 1.08). Increased trans fatty acid intake in women increased the risk of disease (highest vs lowest fifth, HR 2.18, 95\% CI 1.21 to 3.92, per fifth increase HR 1.17, 95\% CI 1.02 to $1.35, p=0.023$ ), with no effect in men (trend across fifths HR 1.01, 95\% CI 0.84 to 1.23$)$.

Conclusion In women, low dietary cholesterol and high trans fatty acid intake increased the risk of developing symptomatic gallstones, for which there are plausible biological mechanisms. No effects were found in men, though this could be due to a lack of power with only half the number of cases diagnosed. Dietary cholesterol and trans fatty acids should be measured in future aetiological studies of gallstones.

Competing interests None.

Keywords aetiology, cholesterol, gallstones, trans fatty acid.

\section{PTH-073 THE ROLE OF DIETARY CHOLESTEROL AND TRANSFATTY ACIDS IN THE AETIOLOGY OF GALLSTONES: A UK PROSPECTIVE COHORT STUDY (EPIC-NORFOLK) USING 7-DAY FOOD DIARIES}

doi:10.1136/gut.2011.239301.474

P J R Banim, ${ }^{1, *}$ R Luben, ${ }^{2}$ S J Sharp, ${ }^{3} \mathrm{~N}$ J Wareham, ${ }^{3} \mathrm{~K}$-T Khaw, ${ }^{2}$ A R Hart ${ }^{1}{ }^{1}$ School of Medicine, University of East Anglia, Norwich, UK; ${ }^{2}$ Institute of Public Health, University of Cambridge, Cambridge, UK; ${ }^{3}$ MRC Epidemiology, University of Cambridge, Cambridge, UK

Introduction Dietary cholesterol may prevent gallstone formation by upregulating the enzyme cholesterol 7 - $\alpha$ hydroxlase (Cyp7A1), which converts cholesterol to primary bile acids and prevents biliary cholesterol supersaturation, a prerequisite for cholesterol stone formation. Trans fatty acids promote hyperinsulinaemia which inhibits Cyp7A1 and could promote gallstone disease. This study investigated dietary cholesterol and trans fatty acid intake on the risk of developing symptomatic gallstones, for the first time using 7-day food diaries (7-DFDs), the most accurate feasible method of estimating food intake in large studies.

Methods 25639 participants (56\% women), aged 40-74 years were recruited into the European Prospective Investigation into Cancer-Norfolk (EPIC-Norfolk) with 23658 completing 7-DFDs at enrolment. The cohort was monitored for 14 years for new symptomatic gallstones, with diagnoses confirmed by review of the clinical notes. The 7-DFDs recorded 1 week's 